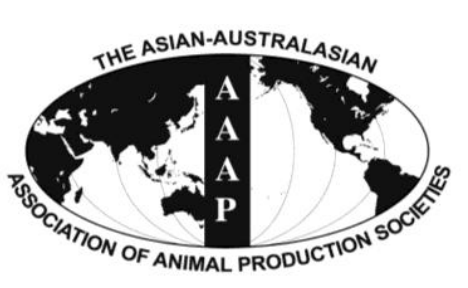

Open Access

Asian Australas. J. Anim. Sci.

Vol. 27, No. 10 : 1461-1468 October 2014

http://dx.doi.org/10.5713/ajas.2013.13629

www.ajas.info

pISSN 1011-2367 elSSN 1976-5517

\title{
Investigation on Antibacterial and Antioxidant Activities, Phenolic and Flavonoid Contents of Some Thai Edible Plants as an Alternative for Antibiotics
}

\author{
J. H. Lee, S. Cho, H. D. Paik ${ }^{1}$, C. W. Choi ${ }^{2}$, K. T. Nam $^{3}$, S. G. Hwang ${ }^{3}$, and S. K. Kim* \\ Department of Animal Science and Technology, Konkuk University, Seoul 143-701, Korea
}

\begin{abstract}
This study was aimed to examine the antibacterial and antioxidative properties of seven edible plants from Thailand to develop alternative antibiotics as feed additives. The plants include Citrus aurantifolia Swingle (Lime) fruits and its leaves, Sesbania grandiflora L. (Agati sesbania) leaves, Piper sarmentosum Roxb (Wild betal) leaves, Curcuma domestica Valeton (Turmeric) roots, Morinda citrifolia L. (Beach mulberry) leaves, Cassia siamea britt (Siamea cassia) leaves, and Cocos nucifera L. (Coconut) peels. The plants were extracted by methanol, $n$-hexane, chloroform, ethyl acetate, butanol and water. Antibacterial activities with minimum inhibitory concentration (MIC) were determined by agar diffusion assay against Escherichia coli, Burkholderia sp., Haemopilus somnus, Haemopilus parasuis, and Clostridium perfringens that were considered pathogenic strains in livestock infection. Methanol extracts of C. aurantifolia Swingle fruits and leaves showed the broadest spectrum of antibacterial activities except for $C$. perfringens. Butanol extract of $S$. grandiflora L. leaves showed the strongest activity against Burkholderia sp. with MIC, $135 \mu \mathrm{g} / \mathrm{mL}$. P. sarmentosum Roxb leaves showed antibacterial activities against $E$. coli, Burkholderia sp. and $H$. parasuis. Ethyl acetate and water extracts from $C$. domesitca Valeton roots showed MIC of $306 \mu \mathrm{g} / \mathrm{mL}$ and $183 \mu \mathrm{g} / \mathrm{mL}$, respectively against only C. perfringens. Antioxidative activity was determined by 2-diphenyl-2-picryl hydrazyl photometric assay. The methanol extracts of $C$. aurantifolia Swingle fruits and $P$. sarmentosum Roxb leaves showed the highest antioxidant activity among all the extracts with $3.46 \mathrm{mg} / \mathrm{mL}$ and $2.70 \mathrm{mg} / \mathrm{mL}$ effective concentration $50 \%\left(\mathrm{EC}_{50}\right)$ values, respectively. Total contents of phenolics and flavonoids were measured from the plant extracts. Methanol extracts of $S$. grandiflora L. and chloroform extracts of $C$. domestica Valeton were found to have the highest amount of total phenolics, 41.7 and $47.8 \mu \mathrm{g} / \mathrm{mL}$, respectively. Flavonoid content of methanol extracts in S. grandiflora $\mathrm{L}$. T was $22.5 \mu \mathrm{g} / \mathrm{mL}$ and the highest among plant extracts tested. These results indicated that $C$. aurantifolia Swingle, S. grandiflora L., P. sarmentosum Roxb, and $C$. domestica Valeton have antibacterial and antioxidant activities and can be used as alternative antibiotics or potential feed additives for the control of animal pathogenic bacteria. (Key Words: Plant Extract, Antibacterial Activity, Antioxidant Activity, Polyphenol, Flavonoid)
\end{abstract}

\section{INTRODUCTION}

Natural plant compounds that can substitute the use of

\footnotetext{
* Corresponding Author: Soo-Ki Kim. Tel: +82-2-450-3728, Fax: +82-2-458-3728, E-mail: sookikim@konkuk.ac.kr

${ }^{1}$ Department of Food Science and Biotechnology of Animal Resources, Konkuk University, Seoul 143-701, Korea.

2 Department of Biology and Medicinal Science, Paichai University, Daejeon 302-735, Korea.

${ }^{3}$ Division of Animal Life and Environmental Science, Hankyong National University, Anseong 456-749, Korea.

Submitted Oct. 5, 2013; Revised Jan. 24, 2014; Accepted May 1, 2014
}

antibiotics have been emerged from health threat of residual antibiotics and outcome of multi-drug resistant pathogenic bacteria in food including animal products. Various plant products in different area were tested and reported as antimicrobial agents and bioactive natural products (Cowan, 1999; Gautam et al., 2007; Magadula and Erasto, 2009). The plants showing antioxidant activity were also known in many reports (Wolfe et al., 2003; Kaur et al., 2006; Ordon et al., 2006; Alviano et al., 2008; Doddola et al., 2008).

The regulation has been reinforced and use of antibiotics has no scope for promotion of livestock growth. From 2006, in-feed antibiotics used in animal production 
have been banned by the European Union. Recently, South Korea has also banned them from the second half of 2011. For rearing of livestock without chemical antibiotics, feeding of natural products with the function such as antimicrobial agents, antioxidants and immune stimulants should be helpful for animal health. Therefore, alternative antibiotics have been regarded as most important feed additives in animal farming. Specially, plant extracts has received much attention in the research for the development of alternative antibiotics (Esquenazi et al., 2002; Wannissorn et al., 2005). The production amounts of plants are much more abundant in subtropical and tropical countries including Thailand compared with northern hemisphere including South Korea with typical four seasons.

The purpose of this study was to examine antibacterial and antioxidant activities and total contents of phenolics and flavonoids of some edible plants of Thailand and to develop natural feed additive for animal health. The plants, viz. Citrus aurantifolia Swingle (Lime) fruit and leaves, Morinda citrifolia (Beach mulberry) leaves, Sesbania grandiflora L. (Agasti sesbania) leaves, Piper sarmentosum (Wild betel) leaves, Curcuma domestica Valeton (Turmeric) roots, Cassia siamea leaves, and Cocos nucifera L. (Coconut) fruits. Tested pathogens using the plant extracts are related to wasting diseases of livestock and causative to execute great economical loss in animal production. Haemopilus parasuis, Haemopilus somnus, and Burkholderia sp. are known to induce pneumonia (Palzer et al., 2008), respiratory disease (Norcia et al., 2004), and meliodiosis (Sprague and Neubauer, 2004; Millan et al., 2007), respectively. Escherichia coli and Clostridium perfringens are causative agents of diarrhoea in piglet (Hendriksen et al., 2006).

\section{MATERIALS AND METHODS}

\section{Plants and preparation of crude extracts}

The plants studied were $C$. aurantifolia Swingle (Lime) fruits and leaves, M. citrifolia (Beach mulberry) leaves, $S$. grandiflora L. (Agasti sesbania) leaves, P. sarmentosum (Wild betel) leaves, C. domestica Valeton (Turmeric) roots, $C$. siamea leaves, and $C$. nucifera $\mathrm{L}$. (Coconut) peels. The plants were cut into smaller species and air-dried in the shade for a week and finely ground. The materials were supplied by K.-C Shin (Bangkok, Thailand) and authenticated by Chang-Won Choi (Department of Biology and Medicinal Science, Paichai University, South Korea). Voucher specimens were deposited at Animal Resources and Research Center, Konkuk University. A sample of $3 \mathrm{~g}$ was suspended in $27 \mathrm{~mL}$ of methanol and the mixture was agitated in $150 \mathrm{rpm}$ for $6 \mathrm{~h}$. The extract was filtered and evaporated. The remained extract was resuspended with 2
$\mathrm{mL}$ of methanol. Methanol extract $(25 \mu \mathrm{L})$ was used for antibacterial activity. One $\mathrm{mL}$ of methanol extract was mixed with $5 \mathrm{~mL}$ of distilled water and $5 \mathrm{~mL}$ of $n$-hexane. The mixture was vigorously mixed and then phase separation was developed by standing. Upper phase of $n$ hexane layer was evaporated and further solvents extractions were done as same manner of previous step. Used solvents were chloroform, ethyl acetate, and butanol, subsequently as described in Figure 1. All of prepared extracts was stored at $4^{\circ} \mathrm{C}$ until assay was performed. Dry matter content was analyzed by estimation of weight loss during heat dry.

\section{Bacterial strains}

Animal pathogenic bacteria used in this study, $H$. somnus, H. parasuis, Burkholderia sp., E. coli, and $C$. perfringens, are related to respiratory disease and diarrhea of piglet and other animals. All of them were collected from stock of National Veterinary Research Quarantine Service in South Korea.

\section{Paper disc diffusion method}

Antibacterial activities of each six fractions from 7 plants were determined using paper disc diffusion method, described elsewhere (Valsaraj et al., 1997). Each bacterial culture $(0.1 \mathrm{~mL})$ grown in Luria-Bertani broth (LB, Difco) at $37^{\circ} \mathrm{C}$ for $18 \mathrm{~h}$ was suspended in $10 \mathrm{~mL}$ of sterilized $0.8 \%$ agar solution. To prevent solidification of agar and heat damage of bacterial cells, $0.8 \%$ agar solution was maintained at $50^{\circ} \mathrm{C}$ until use. After suspension of test strain, agar solution was poured onto LB agar plate and then allowed to be solidified. Plant extracts $(25 \mu \mathrm{L})$ was put on 6 $\mathrm{mm}$ sterilized paper disk and it was dried for $30 \mathrm{~min}$ to evaporate solvent before bacterial cells placed on suspended

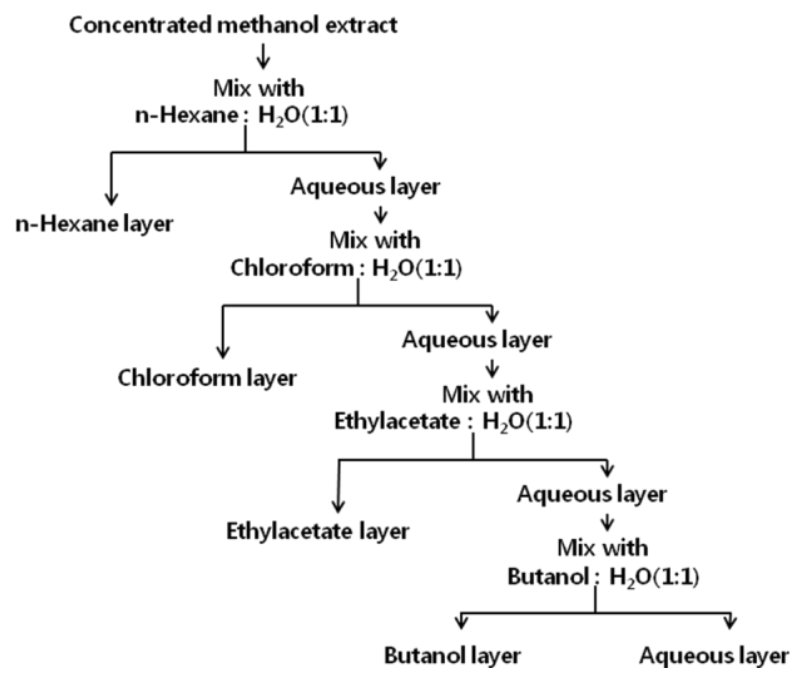

Figure 1. Schematic diagram of plant extract preparation with various solvents. 
agar plate. Prepared test plates were incubated at $37^{\circ} \mathrm{C}$ for $18 \mathrm{~h}$ and then appeared diameters of clear zone were measured.

\section{Determination of minimum inhibitory concentration}

According to the results of screening of antibacterial activities, the minimum inhibitory concentration (MIC) of selected ten plant extracts were determined by serial dilution method on 96 well plates with turbidity measurement (Alviano et al., 2008). Plant extract was diluted with sterilized LB broth, ranged from 0.01 to 10 $\mathrm{mg} / \mathrm{mL}$, and dispensed into 96 well plate. Test strains were inoculated in prepared each well with 1:100 ratio and incubated at $37^{\circ} \mathrm{C}$ for $18 \mathrm{~h}$. Bacterial growth was determined by measuring of turbidity at $600 \mathrm{~nm}$. The MIC was determined as the lowest concentration of extracts which showed no visible growth of the test strains.

\section{Antioxidant activity assay}

The free radical scavenging activities were determined in vitro using 2-diphenyl-2-picryl hydrazyl (DPPH) photometric assay (Tadic et al., 2008) with some modification. Each extract ( 1 to $10 \mu \mathrm{L}$ ) was mixed into its solvent to make a total volume of $100 \mu \mathrm{L}$. It was mixed with $900 \mu \mathrm{L}$ of $0.04 \mathrm{mg} / \mathrm{mL}$ DPPH in methanol solution and the mixture was reacted for $30 \mathrm{~min}$ with gentle agitation. Reduction of violet color induced by free radical scavenging activity was estimated spectrophotometrically at $517 \mathrm{~nm}$. Each solvent used in extraction was applied as negative control and tert-butyl hydroxytoluene was used as positive control. The inhibition percentage was calculated by comparing absorbance with negative control and it was plotted against the concentration of the samples. The extract concentration that represented $50 \%$ of activity was calculated by linear regression analysis and expressed as $\mathrm{EC}_{50}$.

\section{Determination of total polyphenols}

Total polyphenol contents in the extracts were determined by the modified Folin-Ciocalteu method (Wolfe et al., 2003). Each sample $(0.1 \mathrm{~mL})$ was added into $2 \mathrm{~mL}$ of $2 \% \mathrm{Na}_{2} \mathrm{CO}_{3}$. After the tubes were vortexed for $10 \mathrm{~min}$ at room temperature, $0.1 \mathrm{~mL}$ of $50 \%$ Folin-Ciocalteu reagent was added. The tubes were vortexed for $15 \mathrm{~s}$ and allowed to stand for $30 \mathrm{~min}$ at $40^{\circ} \mathrm{C}$ for color development. Absorbance was then measured at $700 \mathrm{~nm}$ using the Shimadzu UV-1601 spectrophotometer. Samples of extracts were evaluated at a final concentration of $1 \mathrm{mg} / \mathrm{mL}$. Total polyphenol contents were calculated as quercetin (25 to 500 ppm) using the following equation based on the calibration curve: $y=0.0033 x-0.0111, R^{2}=0.9979$, where $x$ was the absorbance and $y$ was the quercetin equivalent $(\mu \mathrm{g} / \mathrm{mL})$ (Kiselova et al., 2006).

\section{Determination of total flavonoids}

Total flavonoids were estimated using the method (Ordon et al., 2006). Each sample $(0.5 \mathrm{~mL})$ was added and mixed into $1.5 \mathrm{~mL}$ of $95 \%$ ethanol. To it, $0.1 \mathrm{~mL}$ of $10 \%$ aluminum chloride, $0.1 \mathrm{~mL}$ of $1 \mathrm{M}$ potassium acetate and $2.8 \mathrm{~mL}$ of distilled water were added. After the tubes were mixed for $30 \mathrm{~min}$ at room temperature, the absorbance was measured at $415 \mathrm{~nm}$. A yellow color indicated the presence of flavonoids. Extracts were evaluated at a final concentration of $1 \mathrm{mg} / \mathrm{mL}$. Total flavonoid content was calculated as quercetin ( 5 to $100 \mathrm{ppm}$ ) using the following equation based on the calibration curve: $\mathrm{y}=0.0068 \mathrm{x}-$ $0.0165, R^{2}=0.9974$, where $x$ was the absorbance and $y$ was the quercetin equivalent $(\mu \mathrm{g} / \mathrm{mL})$.

\section{RESULTS}

\section{Antibacterial activity assay}

Methanol extracts of $C$. aurantifolia Swingle fruits showed the most broad spectrum antibacterial activity compared to other solvent extracts, except for $C$. perfringens (Table 1). Butanol and water fractions of it also showed very high antibacterial activities against $H$. somnus, related to porcine respiratory disease causative to wasting syndrome in young pigs. Antibacterial activities of $C$. aurantifolia Swingle leaves appeared different spectrum compared to its fruits. Methanol extract of leaves showed the antibacterial activities against both Burkholderia sp. and H. somnus, but not against E. coli, H. parsuis, and $C$. perfringens. However, the $n$-hexane and chloroform extracts from leaves showed antibacterial activities for Burkholderia sp. only whereas its fruits did not. Only butanol fraction of $S$. grandiflora leaves showed antibacterial activity against Burkholderia sp. related to melioidosis in pig, even in human. Only methanol extracts of $P$. sarmentosum leaves showed very mild antibacterial activities against both E. coli and Burkholderia sp. and moderate activity against $H$. parasuis. Antibacterial spectrum of ethyl acetate and water extracts from $C$. domestica Valeton (Turmeric) roots showed moderate to strong against $C$. perfringens, only. M. citrifolia L. leaves, C. siamea britt leaves, and C. nucifera $\mathrm{L}$. fruits did not show any antibacterial activity against the tested pathogens in this study.

The results of the MIC were well agreed with the antibacterial patterns shown by paper disc diffusion method (Table 2). The strong activity (MIC $495 \mu \mathrm{g} / \mathrm{mL}$ ) was shown by methanol extracts of $C$. aurantifolia Swingle fruits against $H$. somnus. Its leaves showed much lower MIC (198 $\mu \mathrm{g} / \mathrm{mL}$ ) by chloroform extracts than methanol (281 $\mu \mathrm{g} / \mathrm{mL})$ and $n$-hexane $(810 \mu \mathrm{g} / \mathrm{mL})$ extracts against Burkholdeira sp. Butanol extracts of $S$. grandiflora L. showed antibacterial specificity to Burkholdeira sp. with 
Table 1. Antibacterial activities against animal pathogenic bacteria by various plant extracts

\begin{tabular}{|c|c|c|c|c|c|c|c|c|}
\hline \multirow{2}{*}{$\begin{array}{l}\text { Plant tested } \\
\text { (Common name) } \\
\text { (Thailand local name) }\end{array}$} & \multirow{2}{*}{ Part used } & \multirow{2}{*}{ Pathogenic bacteria } & \multicolumn{6}{|c|}{ Solvent fraction $^{1}$} \\
\hline & & & M & $\mathrm{H}$ & $\mathrm{C}$ & $\mathrm{E}$ & B & W \\
\hline \multirow{10}{*}{$\begin{array}{l}\text { Citrus aurantifolia } \text { Swingle } \\
\text { (Lime) } \\
\text { (Manaao) }\end{array}$} & \multirow[t]{5}{*}{ Fruits } & Escherichia coli & ++ & - & - & - & - & - \\
\hline & & Burkholderia sp. & ++ & - & - & - & - & + \\
\hline & & Haemopillus somnus & +++ & - & - & - & +++ & +++ \\
\hline & & Haemopillus parsuis & ++ & - & - & - & - & - \\
\hline & & Clostridium perfringens & - & - & - & - & - & - \\
\hline & \multirow[t]{5}{*}{ Leaves } & Escherichia coli & - & - & - & - & - & - \\
\hline & & Burkholderia sp. & +++ & + & +++ & - & - & - \\
\hline & & Haemopillus somnus & ++ & - & - & - & - & - \\
\hline & & Haemopillus parsuis & - & - & - & - & - & - \\
\hline & & Clostridium perfringens & - & - & - & - & - & - \\
\hline \multirow{5}{*}{$\begin{array}{l}\text { Sesbania grandiflora L. } \\
\text { (Agati sesbania) } \\
\text { (Khaeban or Baikae) }\end{array}$} & \multirow[t]{5}{*}{ Leaves } & Escherichia coli & - & - & - & - & - & - \\
\hline & & Burkholderia sp. & - & - & - & - & +++ & - \\
\hline & & Haemopillus somnus & - & - & - & - & - & - \\
\hline & & Haemopillus parsuis & - & - & - & - & - & - \\
\hline & & Clostridium perfringens & - & - & - & - & - & - \\
\hline \multirow{5}{*}{$\begin{array}{l}\text { Piper sarmentosum Roxb } \\
\text { (Wild betal) } \\
\text { (Chaphulu or Baiphulu) }\end{array}$} & \multirow[t]{5}{*}{ Leaves } & Escherichia coli & + & - & - & - & - & - \\
\hline & & Burkholderia sp. & + & - & - & - & - & - \\
\hline & & Haemopillus somnus & - & - & - & - & - & - \\
\hline & & Haemopillus parsuis & ++ & - & - & - & - & - \\
\hline & & Clostridium perfringens & - & - & - & - & - & - \\
\hline \multirow{5}{*}{$\begin{array}{l}\text { Curcuma domestica } \text { Valeton } \\
\text { (Turmeric) } \\
\text { (Khamin) }\end{array}$} & \multirow[t]{5}{*}{ Roots } & Escherichia coli & - & - & - & - & - & - \\
\hline & & Burkholderia sp. & - & - & - & - & - & - \\
\hline & & Haemopillus somnus & - & - & - & - & - & - \\
\hline & & Haemopillus parsuis & - & - & - & - & - & - \\
\hline & & Clostridium perfringens & - & - & - & ++ & - & +++ \\
\hline \multirow{5}{*}{$\begin{array}{l}\text { Morinda citrifolia } \mathrm{L} . \\
\text { (Beach mulberry) } \\
\text { (Yaeyai or Baiyae) }\end{array}$} & \multirow[t]{5}{*}{ Leaves } & Escherichia coli & - & - & - & - & - & - \\
\hline & & Burkholderia sp. & - & - & - & - & - & - \\
\hline & & Haemopillus somnus & - & - & - & - & - & - \\
\hline & & Haemopillus parsuis & - & - & - & - & - & - \\
\hline & & Clostridium perfringens & - & - & - & - & - & - \\
\hline \multirow{5}{*}{$\begin{array}{l}\text { Cassia siamea britt } \\
\text { (Siamea cassia) } \\
\text { (Kheelek) }\end{array}$} & \multirow[t]{5}{*}{ Leaves } & Escherichia coli & - & - & - & - & - & - \\
\hline & & Burkholderia sp. & - & - & - & - & - & - \\
\hline & & Haemopillus somnus & - & - & - & - & - & - \\
\hline & & Haemopillus parsuis & - & - & - & - & - & - \\
\hline & & Clostridium perfringens & - & - & - & - & - & - \\
\hline \multirow{5}{*}{$\begin{array}{l}\text { Cocos nucifera } \mathrm{L} \\
\text { (Coconut) } \\
\text { (Maphraogere) }\end{array}$} & \multirow[t]{5}{*}{ Peels } & Escherichia coli & - & - & - & - & - & - \\
\hline & & Burkholderia sp. & - & - & - & - & - & - \\
\hline & & Haemopillus somnus & - & - & - & - & - & - \\
\hline & & Haemopillus parsuis & - & - & - & - & - & - \\
\hline & & Clostridium perfringens & - & - & - & - & - & - \\
\hline
\end{tabular}

$\mathrm{M}$, methanol; $\mathrm{H}, n$-hexane; $\mathrm{C}$, chloroform; E, ethyl acetate; $\mathrm{B}$, butanol; $\mathrm{F}$, water.

${ }^{1}$ Antibacterial activities: -, no inhibition ( $\left.8 \mathrm{~mm}\right) ;+$, very mild inhibition (9 to $\left.11 \mathrm{~mm}\right) ;++$, moderate inhibition (11 to $13 \mathrm{~mm}$ ); +++, strong inhibition (13 to $17 \mathrm{~mm}$ ).

MIC of $135 \mu \mathrm{g} / \mathrm{mL}$. Methanol extracts of Piper against only $C$. perfringens with $306 \mu \mathrm{g} / \mathrm{mL}$ and 183 sarmentosum Roxb had MICs, $502 \mu \mathrm{g} / \mathrm{mL}, 1,005 \mu \mathrm{g} / \mathrm{mL}, \mu \mathrm{g} / \mathrm{mL}$ MICs, respectively. and $251 \mu \mathrm{g} / \mathrm{mL}$ against E. coli, Burkholdeira sp., and $H$. parasuis, respectively. The ethyl acetate and water extracts from $C$. domesitca Valeton roots have high specificities

\section{Antioxidant activity assay}

C. aurantifolia Swingle fruit showed relatively high 
Table 2. Minimum inhibitory concentration against animal pathogenic bacteria by various plant extracts

\begin{tabular}{|c|c|c|c|c|c|c|c|}
\hline \multirow[b]{2}{*}{ Plant tested } & \multirow[b]{2}{*}{ Part used } & \multirow[b]{2}{*}{ Fraction } & \multicolumn{5}{|c|}{ Pathogenic bacteria } \\
\hline & & & $\begin{array}{c}\text { Escherichia } \\
\text { coli }\end{array}$ & $\begin{array}{c}\text { Burkholderia } \\
\text { sp. }\end{array}$ & $\begin{array}{c}\text { Haemopillus } \\
\text { somnus }\end{array}$ & $\begin{array}{c}\text { Haemopillus } \\
\text { parasuis }\end{array}$ & $\begin{array}{l}\text { Clostridium } \\
\text { perfringens }\end{array}$ \\
\hline \multirow{7}{*}{$\begin{array}{l}\text { Citrus aurantifolia } \\
\text { Swingle }\end{array}$} & \multirow{4}{*}{ Fruits } & & ------------- & -- Minimum in & hibitory concel & tration $(\mu \mathrm{g} / \mathrm{mL}$ & 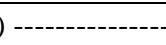 \\
\hline & & Methanol & 1,980 & 990 & 495 & 990 & $\mathrm{ND}^{1}$ \\
\hline & & Butanol & ND & ND & 660 & ND & ND \\
\hline & & Water & ND & 660 & 660 & ND & ND \\
\hline & \multirow[t]{3}{*}{ Leaves } & Methanol & ND & 281 & 2,250 & ND & ND \\
\hline & & $n$-Hexane & ND & 810 & ND & ND & ND \\
\hline & & Chloroform & ND & 198 & ND & ND & ND \\
\hline Sesbania grandiflora $\mathrm{L}$. & Leaves & Butanol & ND & 135 & ND & ND & ND \\
\hline Piper sarmentosum Roxb & Leaves & Methanol & 502 & 1,005 & ND & 251 & ND \\
\hline \multirow[t]{2}{*}{ Curcuma domestica Valeton } & Roots & Ethyl acetate & ND & ND & ND & ND & 306 \\
\hline & & Water & ND & ND & ND & ND & 183 \\
\hline
\end{tabular}

ND, not detected.

Table 3. Antioxidant activities of used plants extracts

\begin{tabular}{lllr}
\hline \multirow{2}{*}{ Plant tested } & Part used & \multicolumn{2}{c}{ Free radical scavenging activity } \\
\cline { 3 - 4 } Citrus aurantifolia Swingle & Fruits & Methanol & EC $_{50}$ value $^{1}\left(\mathrm{mg} / \mathrm{mL}^{\prime}\right)$ \\
& & Butanol & $3.46 \pm 0.2$ \\
& & Water & $8.89 \pm 1.2$ \\
& Leaves & Methanol & $12.16 \pm 0.6$ \\
& & $n$-Hexane & $17.68 \pm 7.4$ \\
& & Chloroform & $10.27 \pm 4.6$ \\
Sesbania grandiflora L. & Leaves & Butanol & ND \\
Piper sarmentosum Roxb & Leaves & Methanol & $2.70 \pm 0.3$ \\
Curcuma domestica Valeton & Roots & Ethyl acetate & ND \\
& & Water & $18.18 \pm 0.8$ \\
\hline
\end{tabular}

ND, not detected.

${ }^{1} \mathrm{EC}_{50}$ value means the concentration at $50 \%$ of antioxidant activity. In the case of positive control determined by $\mathrm{BHT}$, EC $\mathrm{E}_{50} \mathrm{was} 0.3 \pm 0.04 \mathrm{mg} / \mathrm{mL}$

antioxidant activity (Table 3), specifically in methanol extract $\left(\mathrm{EC}_{50}, 3.46 \mathrm{mg} / \mathrm{mL}\right)$. Antioxidant activity of chloroform extract of $C$. aurantifolia Swingle leaves was higher than that $\left(\mathrm{EC}_{50}, 10.27 \mathrm{vs} 17.68 \mathrm{mg} / \mathrm{mL}\right)$ of methanol extract. However, no activity was detected by $n$-hexane extract although it showed antibacterial activity (Tables 1 and 2). Butanol extract of Sesbania grandiflora L. leaves showed no antioxidant activity. On the other hand, $P$. sarmentosum Roxb showed the most strong antioxidant activity with $\mathrm{EC}_{50}, 2.70 \mathrm{mg} / \mathrm{mL}$. Water extracts of $C$. domestica Valeton roots showed the moderate antioxidant activity $\left(\mathrm{EC}_{50}, 18.18 \pm 0.8 \mathrm{mg} / \mathrm{mL}\right)$ whereas ethyl acetate fraction did not.

\section{Total polyphenolic compounds and flavonoids}

Among the plant extracts tested in the study, methanol extracts of $S$. grandiflora L. and chloroform extracts of $C$. domestica Valeton were found to have the highest amount of total polyphenol, 41.7 and $47.8 \mu \mathrm{g} / \mathrm{mL}$, respectively
(Table 4). The highest amount of total flavonoid recorded among all plant extracts tested was $22.5 \mu \mathrm{g} / \mathrm{mL}$, which was also from methanol extract of $S$. grandiflora L. Methanol extracts of $P$. sarmentosum Roxb showed high content $(19.4 \pm 0.008 \mu \mathrm{g} / \mathrm{mL})$ of total flavonoid. Most of the methanol extracts of tested plants showed high amounts of total polyphenol and total flavonoid compared to other solvent extracts except for Curcuma domestica, where chloroform extract showed highest (Table 4).

\section{DISCUSSION}

In this study, 6 kinds of solvent extraction were applied to 7 edible plants of Thailand and their antibacterial activities were determined by the spectrum against 5 animal pathogenic bacteria. Some bioactive components of these plants may differ in their solubility depending on the extractive solvents used. With these results, finally 10 extracts from 4 plants were selected and their MICs and 
Table 4. Total polyphenolics and flavonoids in tested plants extracts

\begin{tabular}{|c|c|c|c|c|}
\hline \multirow{2}{*}{ Plant tested } & \multirow{2}{*}{ Part used } & \multirow{2}{*}{ Solvent fraction } & \multicolumn{2}{|c|}{ Contents $(\mu \mathrm{g} / \mathrm{mL})^{1}$} \\
\hline & & & Total polyphenol & Total flavonoid \\
\hline \multirow[t]{12}{*}{ Citrus aurantifolia Swingle } & \multirow[t]{6}{*}{ Fruits } & Methanol & $28.5 \pm 0.004$ & $15.8 \pm 0.007$ \\
\hline & & $n$-Hexane & $18.2 \pm 0.009$ & $9.4 \pm 0.005$ \\
\hline & & Chloroform & $24.1 \pm 0.004$ & $9.7 \pm 0.012$ \\
\hline & & Ethyl acetate & $13.6 \pm 0.006$ & $8.8 \pm 0.006$ \\
\hline & & Butanol & $20.4 \pm 0.009$ & $10.0 \pm 0.006$ \\
\hline & & Water & $12.6 \pm 0.002$ & $11.0 \pm 0.013$ \\
\hline & \multirow[t]{6}{*}{ Leaves } & Methanol & $31.8 \pm 0.003$ & $17.5 \pm 0.003$ \\
\hline & & $n$-Hexane & $18.6 \pm 0.004$ & $5.9 \pm 0.007$ \\
\hline & & Chloroform & $14.5 \pm 0.003$ & $5.8 \pm 0.008$ \\
\hline & & Ethyl acetate & $11.3 \pm 0.002$ & $4.4 \pm 0.002$ \\
\hline & & Butanol & $22.9 \pm 0.005$ & $8.8 \pm 0.013$ \\
\hline & & Water & $9.8 \pm 0.005$ & $5.8 \pm 0.008$ \\
\hline \multirow[t]{6}{*}{ Sesbania grandiflora L. } & \multirow[t]{6}{*}{ Leaves } & Methanol & $41.7 \pm 0.006$ & $22.5 \pm 0.006$ \\
\hline & & $n$-Hexane & $20.7 \pm 0.008$ & $7.7 \pm 0.006$ \\
\hline & & Chloroform & $26.0 \pm 0.004$ & $7.0 \pm 0.013$ \\
\hline & & Ethyl acetate & $11.0 \pm 0.005$ & $10.0 \pm 0.014$ \\
\hline & & Butanol & $11.3 \pm 0.004$ & $9.0 \pm 0.020$ \\
\hline & & Water & $17.4 \pm 0.006$ & $7.0 \pm 0.013$ \\
\hline \multirow[t]{6}{*}{ Piper sarmentosum Roxb } & \multirow[t]{6}{*}{ Leaves } & Methanol & $38.8 \pm 0.006$ & $19.4 \pm 0.008$ \\
\hline & & $n$-Hexane & $17.6 \pm 0.009$ & $10.2 \pm 0.012$ \\
\hline & & Chloroform & $23.3 \pm 0.010$ & $9.2 \pm 0.018$ \\
\hline & & Ethyl acetate & $13.5 \pm 0.013$ & $5.2 \pm 0.010$ \\
\hline & & Butanol & $23.9 \pm 0.007$ & $11.3 \pm 0.011$ \\
\hline & & Water & $17.7 \pm 0.006$ & $4.4 \pm 0.006$ \\
\hline \multirow[t]{6}{*}{ Curcuma domestica Valeton } & \multirow[t]{6}{*}{ Roots } & Methanol & $25.9 \pm 0.004$ & $14.1 \pm 0.002$ \\
\hline & & $n$-Hexane & $24.5 \pm 0.009$ & $7.5 \pm 0.010$ \\
\hline & & Chloroform & $47.8 \pm 0.008$ & $14.1 \pm 0.012$ \\
\hline & & Ethyl acetate & $19.7 \pm 0.003$ & $5.8 \pm 0.008$ \\
\hline & & Butanol & $18.5 \pm 0.004$ & $7.0 \pm 0.015$ \\
\hline & & Water & $27.1 \pm 0.011$ & $12.7 \pm 0.006$ \\
\hline
\end{tabular}

${ }^{1}$ Total polyphenol and flavonoid contents were calculated as quercetin equivalent.

antioxidant activities were determined. As a result, $C$. aurantifolia Swingle fruits known as lime showed the most broad antibacterial spectrum with the strong antioxidant activity specially in methanol extracts. It has been traditionally regarded as an effective carminative fruit in Thailand and the antibacterial activity of its essential oil has been reported against Salmonella typhimurium, $S$. enteritidis, E. coli, C. perfringens, and Campylobacter jejuni (Wannissorn et al., 2005). In this study, we newly found that the fruit extracts of $C$. aurantifolia Swingle also had the antibacterial activities against Burkholderia sp., $H$. somnus, and $H$. parasuis including E. coli. However, we did not find the antibacterial activity from the fruit extracts of $C$. aurantifolia Swingle against $C$. perfringens. There may be a difference in concentration of effective compounds between essential oil and our solvent extraction. C. aurantifolia Swingle leaves contained more strong antimicrobial activity against Burkholderia sp. (Tables 1 and 2) compared to its fruits. Antibacterial properties of different parts in $C$. aurantifolia Swingle were reported against Gram-positive bacteria such as Staphylococcus aureus, Gram-negative bacteria including E. coli, S. paratyphi, Pseudomonas aeruginosa, and Candida albicans (Aibinu et al., 2007). Patil et al. (2009) reported that the chloroform extracts of $C$. aurantifolia Swingle fruit had much higher free radicalscavenging activity than acetone and methanol extracts. Immunomodulatory effect of concentrated lime juice extract was also observed on activated human mononuclear cells (Gharagozloo et al., 2001).

Although a few suggestions about antibacterial activity against skin and gastrointestinal microbes have been reported (Denyer, 1990; Doddola et al., 2008), there is little information about antimicrobial activities of $S$. grandiflora L. leaves. Antibacterial activity against Burkholderia sp. 
was found (Tables 1 and 2) in butanol fraction of $S$. grandiflora L. leaves. Zaidan et al. (2005) reported the antibacterial activities of methanol extracts of $P$. sarmentosum leaves against $S$. aureus and $P$. aeruginosa. In this study, $P$. sarmentosum Roxb also showed the antibacterial activities and the highest antioxidant activity, especially, its methanol extracts showed the antibacterial activities against $E$. coli, Burkholderia sp. and $H$. parasuis. C. domestica Valeton roots have been widely cultivated in Southeast Asia for the use of an additive in cooking and a traditional medicine to treat diarrhea (Ahmad-Raus et al., 2001). Interestingly, antibacterial spectrum of ethyl acetate and water extracts from $C$. domestica Valeton roots showed specificities against $C$. perfringens (Tables 1 and 2), in contrary to the report of Wannissorn et al. (2005).

M. citrifolia L. is widely grown in the Hawaii and Asia and it has been commonly called as "Noni". It has been used as an herbal plant with effectiveness on diabetes, hypertension and immunomodulation (Takashima et al., 2007). Schäfer et al. (2008) showed the bactericidal effect against Escherichia coli in calves fed M. citrifolia (Noni) puree. Blood samples from Noni puree-fed calves displayed significantly more bacterial killing against $E$. coli than did controls on day 14. C. siamea britt is commonly found tree in Southeast Asia and its bark, root and flowers have been known to have medicinal values in diabetes, hypertension and insomnia (Kaur et al., 2006). Methanol extracts of $C$. siamea exhibited antibacterial activities against both Bacillus cereus and Listeria monocytogenes whereas $M$. citrifolia L. had no antibacterial activities for all tested microorganisms (Nanasombat and Teckchuen, 2009). In this study, the antibacterial activities of $M$. citrifolia L., $C$. siamea britt and $C$. nuricifera $\mathrm{L}$. were not detected against tested pathogens. Husk of $C$. nuricifera $\mathrm{L}$. fruits have various phenolic compounds that have the physiological effects including antileishmanial, antibacterial, antiviral and anti-neoplasic (Alviano et al., 2008). In addition, Esquenazi et al. (2002) reported that extracts of $C$. nuricifera showed the antibacterial activity against $S$. aureus. In an earlier study (Wannissorn et al., 2005), the antibacterial activity against $C$. perfringens was reported as very high, however, no activity was found at all of solvent fractions in this study.

Biological activities related to antibacterial and antioxidant activities may be correlated with total polyphenol and flavonoid (Rice-Evans et al., 1997; Cushnie and Lamb, 2005; Daglia, 2011). Even though the correlation was not perfectly matched in this study, methanol extracts of both Citrus aurantifolia Swingle and Piper sarmentosum Roxb possessed high antibacterial and antioxidant activities in proportion to high amounts of total polyphenol and flavonoid. In conclusion, the extracts from Citrus aurantifolia Swingle (Lime), Sesbania grandiflora L. (Agati sesbania), Piper sarmentosum Roxb (Wild betal) and
Curcuma domestica Valeton (Turmeric) showed high antibacterial and antioxidant activities. They should be further tested in vivo animal experiments as alternative antibiotics in potential feed additives for the inhibition of respiratory disease and meliodiosis with diarrhea in livestock.

\section{ACKNOWLEDGMENTS}

Fund for research was provided by Biogreen 21 of Rural Development Administration of Korea. We are thankful to K. C. Shin for providing of air-dried plant materials used in this study.

\section{REFERENCES}

Ahmad-Raus, R. R., E. E. S. Abdul-Latif, and J. J. Mohammad. 2001. Lowering of lipid composition in aorta of guinea pigs by Curcuma domestica. BMC Complement Altern. Med. 1:6-10.

Aibinu, I., T. Adenipekun, T. Adelowotan, T. Ogunsanya, and T. Odugbemi. 2007. Evaluation of the antimicrobial properties of different parts of citrus aurantifolia (lime fruit) as used locally. Afr. J. Tradit. Camplement Altern. Med. 4:185-190.

Alviano, W. S., D. S. Alviano, C. G. Diniz, A. R. Antoniolli, C. S. Alviano, L. M. Farias, M. A. R. Carvalho, M. M. G. Souza, and A. M. Bolognese. 2008. In vitro antioxidant potential of medicinal plant extracts and their activities against oral bacteria based on Brazilian folk medicine. Arch. Oral Biol. 53: 545-552.

Cowan, M. M. 1999. Plant products as antimicrobial agents. Clin. Microbiol. Rev. 12:564-582.

Cushnie, T. P. T., and A. J. Lamb. 2005. Antimicrobial activity of flavonoids. Int. J. Antimicrob. Agents. 26:343-356.

Daglia, M. 2011. Polyphenols as antimicrobial agents. Curr. Opin. Biotechnol. 23:174-181.

Denyer, S. P. 1990. Guide to microbiological control in pharmaceuticals. Ellis Horwood, UK. 11-28.

Doddola, S., H. Pasupulati, B. Kogantia, and K. V. S. R. G. Prasad. 2008. Evaluation of Sesbania grandiflora for antiurolithiatic and antioxidant properties. J. Nat. Med. (Tokyo) 62:300-307.

Esquenazi, D., M. D. Wigg, M. M. F. S. Miranda, H. M. Rodrigues, J. B. F. Tostes, S. Rozental, A. J. R. da Silva, and C. S. Alviano. 2002. Antimicrobial and antiviral activities of polyphenolics from Cocos nucifera Linn. (Palmae) husk fiber extract. Res. Microbiol. 153:647-652.

Gautam, R., A. Saklani, and S. M. Jachak. 2007. Indian medicinal plants as a source of antimycobacterial agents. J. Ethnopharmacol. 110: 200-234.

Gharagozloo, M. and A. Ghaderi. 2001. Immunomodulatory effect of concentrated lime juice extract on activated human mononuclear cells. J. Ethnopharmacol. 77:85-90.

Hendriksen, S. W., L. A. van Leengoed, H. I. Roest, and A. van Nes. 2006. Neonatal diarrhea in pigs: alpha- and beta2-toxin produced by Clostridium perfringens. Tijdschr. Diergeneeskd. 131:910-913.

Kaur, G., M. S. Alam, Z. Jabbar, K. Javed, and M. Athar. 2006. Evaluation of antioxidant activity of Cassia siamea flowers. J. 
Ethnopharmacol. 108:340-348.

Kiselova, Y., D. Ivanova, T. Chervenkov, D. Gerova, B. Galunska, and T. Yankova. 2006. Correlation between the in vitro antioxidant activity and polyphenol content of aqueous extracts from bulgarian herbs. Phytother. Res. 20:961-965.

Magadula, J. J. and P. Erasto. 2009. Bioactive natural products derived from the East African flora. Nat. Prod. Rep. 26:15351554.

Millan, J. M., M. Mayo, D. Gal, A. Janmaat, and B. J. Currie. 2007. Clinical variation in melioidosis in pigs with clonal infection following possible environmental contamination from bore water. Vet. J. 174: 200-202.

Nanasombat, S. and N. Teckchuen. 2009. Antimicrobial, antioxidant and anticancer activities of Thai local vegetables. J. Med. Plants Res. 3:443-449.

Norcia, L. J., A. M. Silvia, S. L. Santoro, J. Retsema, M. A. Letavic, B. S. Bronk, K. M. Lundy, B. Yang, N. A. Evans, and S. F. Hayashi. 2004. In vitro microbiological characterization of a novel azalide, two triamilides and an azalide ketal against bovine and porcine respiratory pathogens. J. Antibiot. (Tokyo) 57:280-288

Ordon, Ez A. A. L., J. D. Gomez, M. A. Vattuone, and M. I. Isla. 2006. Antioxidant activities of Sechium edule (Jacq.) Swart extracts. Food Chem. 97:452-458.

Palzer, A., M. Ritzmann, G. Wolf, and K. Heinritzi. 2008. Associations between pathogens in healthy pigs and pigs with pneumonia. Vet. Rec. 162:267-271.

Patil, J. R., K. N. Chidambara Murthy, G. K. Jayaprakasha, M. B. Chetti, and B. S. Patil. 2009. Bioactive compounds from Mexican lime (Citrus aurantifolia) juice induce apoptosis in human pancreatic cells. J. Agric. Food Chem. 57:10933-10942.
Rice-Evans, C., N. Miller, and G. Paganga. 1997. Antioxidant properties of phenolic compounds. Trends Plant Sci. 2:152-159.

Schäfer, M., P. Sharp, V. J. Brooks, J. Xu, J. Cai, N. S. Keuler, S. F. Peek, R. G. Godbee, R. D. Schultz, and B. J. Darien. 2008. Enhanced bactericidal activity against Escherichia coli in calves fed Morinda citrifolia (Noni) puree. J. Vet. Intern. Med. 22:499-502.

Sprague, L. D. and H. Neubauer. 2004. Melioidosis in animals: a review on epizootiology, diagnosis and clinical presentation. J. Vet. Med. B. 51:305-320.

Tadić, V. M., S. Dobrić, G. M. Marković, S. M. Dordević, I. S. Arsić, N. R. Menković, and T. Stević. 2008. Anti-inflammatory, gastroprotective, free-radical-scavenging, and antimicrobial activities of Hawthorn berries ethanol extract. J. Agric. Food Chem. 56:7700-7709.

Takashima, J., Y. Ikeda, K. Komiyama, M. Hayashi, A. Kishida, and A. Ohsaki. 2007. New constituents from the leaves of Morinda citrifolia. Chem. Pharm. Bull. 55:343-345.

Valsaraj, R., P. Pushpangadan, U. W. Smitt, A. Adsersen, and U. Nyman. 1997. Antimicrobial screening of selected medicinal plant from India. J. Ethnopharmacol. 58:75-83.

Wannissorn, B., S. Jarikasem, T. Siriwangchai, and S. Thubthimthed. 2005. Antibacterial properties of essential oils from Thai medicinal plants. Fitoterapia 76:233-236.

Wolfe, K., X. Wu, and R. H. Liu. 2003. Antioxidant activity of apple peels. J. Agric. Food Chem. 51:609-614.

Zaidan, M. R. S., A. Noor Rain, A. R. Badrul, A. Adlin, A. Norazah, and I. Zakiah. 2005. In vitro screening of five local medicinal plants for antibacterial activity using disc diffusion method. Trop. Biomed. 22:165-170. 